\title{
Predictability of myometrial, lower uterine segment and cervical invasion with 3D transvaginal ultrasonography and magnetic resonance imaging in endometrial cancer patients: a prospective cohort study
}

\author{
Nuri Yildirim¹, Bahadir Saatli ${ }^{2}$, Semir Kose ${ }^{2}$, Ceren Sancar ${ }^{3}$, Cagnur Ulukus ${ }^{4}$, \\ Meral Koyuncuoglu ${ }^{4}$, Ugur Saygili², Funda Obuz ${ }^{5}$
}

${ }^{1}$ Ege University, Faculty of Medicine, Department of Obstetrics and Gynecology, Division of Gynecologic Oncology, ${ }^{2}$ Dokuz Eylul University, Faculty of Medicine, Department of Obstetrics and Gynecology, ${ }^{3}$ Ege University, Faculty of Medicine, Department of Obstetrics and Gynecology, ${ }^{4}$ Dokuz Eylul University, Faculty of Medicine, Department of Pathology, ${ }^{5}$ Dokuz Eylul University, Faculty of Medicine, Department of Radiology, Izmir, Turkey

\begin{abstract}
Aims: The objective of this study is to identify the diagnostic performance of three-dimensional transvaginal ultrasonography (3D-US) and magnetic resonance imaging (MRI) in detecting myometrial, lower uterine segment and/or cervical invasion in endometrial cancer patients. Materials and methods: In this prospective study, 40 patients diagnosed with endometrial cancer were performed 3D-US and MRI, preoperatively. Deep myometrial, lower uterine segment and cervical invasion were evaluated subjectively and results were compared with the final histology as a gold standard. Results: Diagnostic accuracy of 3D-US for detecting deep myometrial, lower uterine segment and cervical invasion were $87.5 \%, 80 \%$ and $85 \%$, respectively. The same results for MRI were $75 \%, 65 \%$ and $70 \%$, respectively. For deep myometrial, lower uterine segment and/or cervical invasion in endometrial cancer, 3D-US had higher sensitivity, specificity, negative and positive predictive value and accuracy than MRI. The combination of these two imaging techniques had an increased sensitivity of detecting all parameters related with tumoral invasion but decreased specificity and the accuracy. Conclusion: 3D-US had better performance in detecting myometrial, lower uterine segment and/or cervical invasion than MRI in endometrial cancer patients. Combination of these techniques was not preferred according to this study.

Keywords: endometrial cancer; 3D transvaginal ultrasonography; magnetic resonance imaging; myometrial invasion; cervical invasion; lower uterine segment invasion
\end{abstract}

\section{Introduction}

Endometrial cancer is the most common gynecologic cancer among developed countries [1]. Surgical staging is the first step for both treatment and determining the prognosis. In the staging procedure, total abdominal

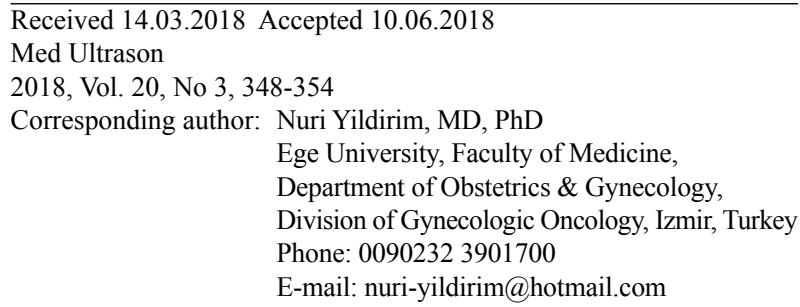

hysterectomy and bilateral salpingo-oohorectomy is the cornerstone. Lymphadenectomy, either pelvic or pelvic/ paraaortic, is performed in advanced stages but in early stages it is controversial [2,3]. Prognostic factors such as histologic type, grade, depth of myometrial invasion and tumor size are important in the decision for lymphadenectomy $[4,5]$. Some of these risk factors directly change the International Federation of Gynecology and Obstetrics (FIGO) stage [6]. Even in low-grade endometrioid tumors, lymph node metastasis risk increases from $4 \%$ to $15 \%$ if the tumor invades more than $50 \%$ of the myometrium [7]. Additionally, according to some data, lower uterine segment invasion (LUSI) in the early stage of endometrial cancer is associated with poor prognostic 
factors such as high grade, deep myometrial invasion and lymphatic spread and it was reported that LUSI should be considered as a high-risk factor [8-10].

Gold standard method for detecting tumoral invasion in endometrial cancer is histopathological evaluation. But especially in type I tumors, preoperative assessment is required in order to discriminate low and high-risk patients for making a decision for further dissection. In endometrial cancer, the most common used imaging technique is transvaginal ultrasonography (TVUS). In a recent metaanalysis, the sensitivity and specificity of TVUS for diagnosing deep myometrial invasion were $75 \%$ and $86 \%$, respectively [11]. Magnetic resonance imaging (MRI) is also preferred in preoperative assessment due to its efficacy in soft tissue evaluation. The sensitivity and specificity of MRI for detecting deep invasion was reported as $83 \%$ and $82 \%$, respectively [11].

Three-dimensional TVUS (3D-US) is a relatively new US technique used in preoperative assessment. It provides an evaluation in three different axes and has an advantage to store the image and examine the data later and repeatedly. In a recent study the sensitivity, specificity and negative predictive value of 3D-US for detecting deep myometrial invasion in endometrial cancer patients were $100 \%, 88 \%$ and $100 \%$, respectively [12].

Up to now, there are only three retrospective studies that compare 3D-US with MRI for detecting deep myometrial invasion in endometrial cancer [13-15]. This is the first study that compares 3D-US and MRI for detecting deep myometrial, lower uterine segment and cervical invasion in endometrial cancer patients in a prospective manner.

\section{Materials and methods}

Forty patients diagnosed with endometrial cancer with endometrial biopsy (dilatation and curettage) between 2013-2016, in Dokuz Eylul University, Faculty of Medicine, Department of Obstetrics and Gynecology, were included in this study. Patients who were clinically in advanced stages or were unsuitable for surgery due to comorbid diseases were excluded. The study protocol was approved by theInstitutional Ethics Committee.

The patients were operated in maximum a two-month period after biopsy. All patients had MRI and 3D-US in a period of 15 days before surgery and were operated via laparotomy with a mid-line incision. First preoperative peritoneal washings were collected for cytological evaluation. Than total abdominal hysterectomy, bilateral salpingo-oophorectomy was performed and the uterus was sent for frozen section. In patients with non-endometrioid histology, cervical invasion, myometrial inva- sion more than $50 \%$ and/or grade-3 tumor (according to preoperative endometrial biopsy and intra-operative frozen section), pelvic and paraaortic lymphadenectomy was performed. In patients with the tumor size more than $2 \mathrm{~cm}$ and lower uterine segment invasion only, a pelvic lymphadenectomy was performed. Lymphadenectomy was not performed in patients with endometrioid histology, superficial myometrial invasion, low-grade tumor and a tumor size less than $2 \mathrm{~cm}$.

The final pathology report was accepted as a gold standard. The combination of 3D-US and MRI was accepted as positive if at least one of them evidenced deep myometrial, cervical and LUSI invasion.

\section{D-US evaluation}

Voluson 730 Expert machine (GE Medical Systems, Zipf, Austria) with an endovaginal probe (RIC5-9H) was used for the US examination of all patients. Once the uterus was scanned to obtain a 3D view, the data was stored on the hard disk (Sonoview, GE Medical Systems, Zipf, Austria) in order to analyze them later. Offline analysis was performed with 4DView Software (4D View 17, ver Ext.1; GE Medical Systems, Zipf, Austria). Examiners were blinded to the MRI and final pathology of the patient and were experienced in 3D-US more than 4 years. (Fig 1 and 2) Sagittal, transverse and coronal sections of the uterus were evaluated at the same screen by sweeping through the scanned 3D uterine view and the decision for myometrial invasion less or more than half of the myometrium was made by the subjective impression of the examiner. In this subjective interpretation, the most suspicious area for myometrial invasion was compared with the endometrial-myometrial junction in the adjacent and opposite sides of the uterus. Similarly, the LUSI and cervical invasion was accepted as positive if the examiner suspected tumoral invasion at these sides, subjectively.

\section{MRI evaluation}

MRI was performed in all patients with 1.5 Tesla scanner (Philips Achieva, Philips Healthcare, Amsterdam, Holland). The examiner was blinded to both final histology and 3D-US results and was experienced in abdominal radiology. Sagital, axial oblique fast spin-echo T2-weighted images and axial oblique fat-saturated T1 sequences including entire pelvis after the administration of gadolinium were obtained. Endo/myo-metrial junction was identified and the presence of disturbed borders was evaluated as invasion of tumor. Presence or absence of deep myometrial invasion was decided according to maximum depth of invasion, subjectively. The LUSI and cervical invasion was accepted as positive if the examiner suspected tumoral invasion at these sides, subjectively. 


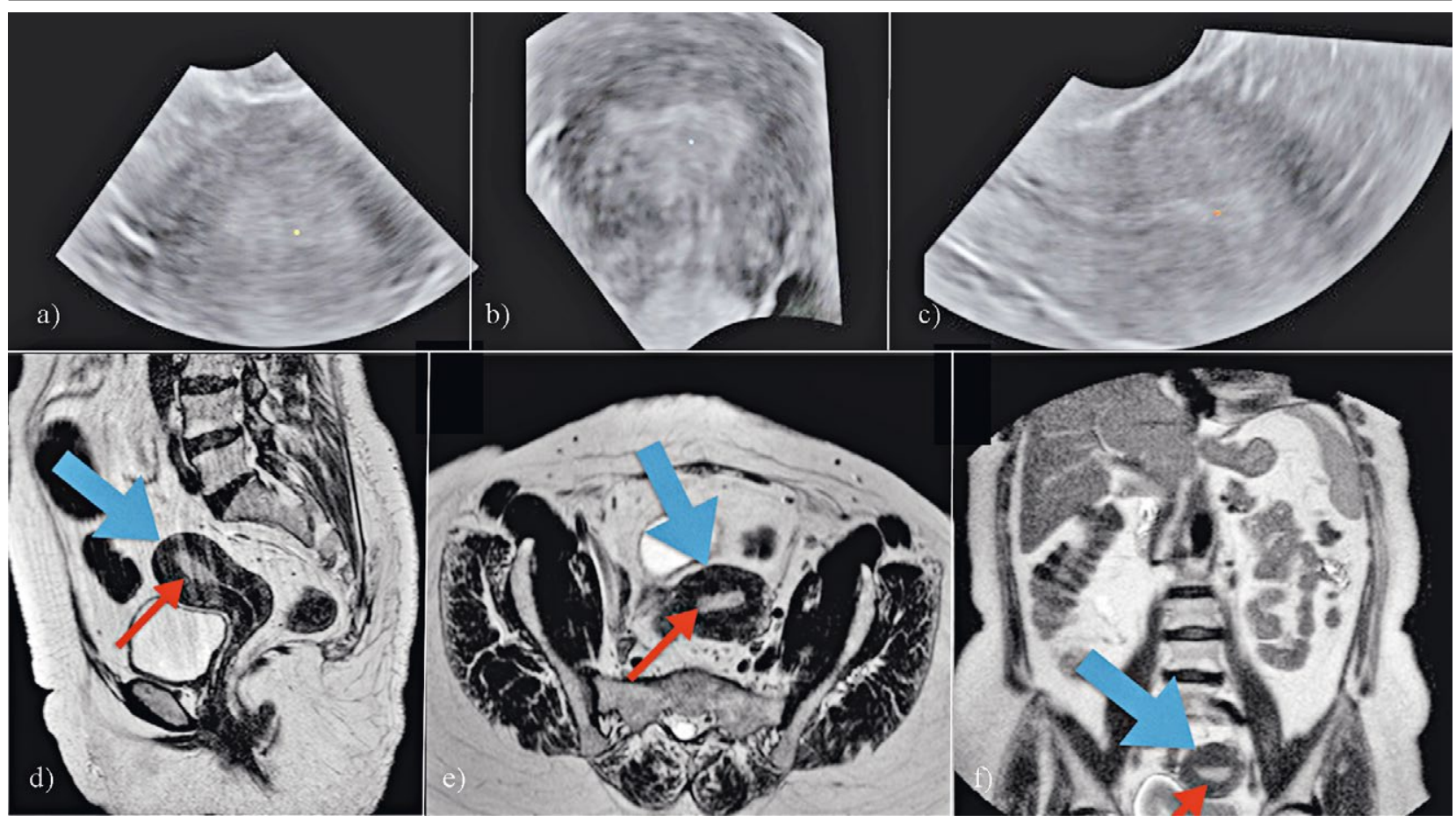

Fig 1. A patient with endometrial cancer without myometrial invasion: 3-dimensional sonographic view [a) transverse, b) sagittal and c) coronal view of uterus] and T2-weighted magnetic resonance [d) sagittal, e) transverse and f) coronal view]. Blue thick arrow indicates uterus. Red thin line indicates the localization of endometrial-myometrial junction.

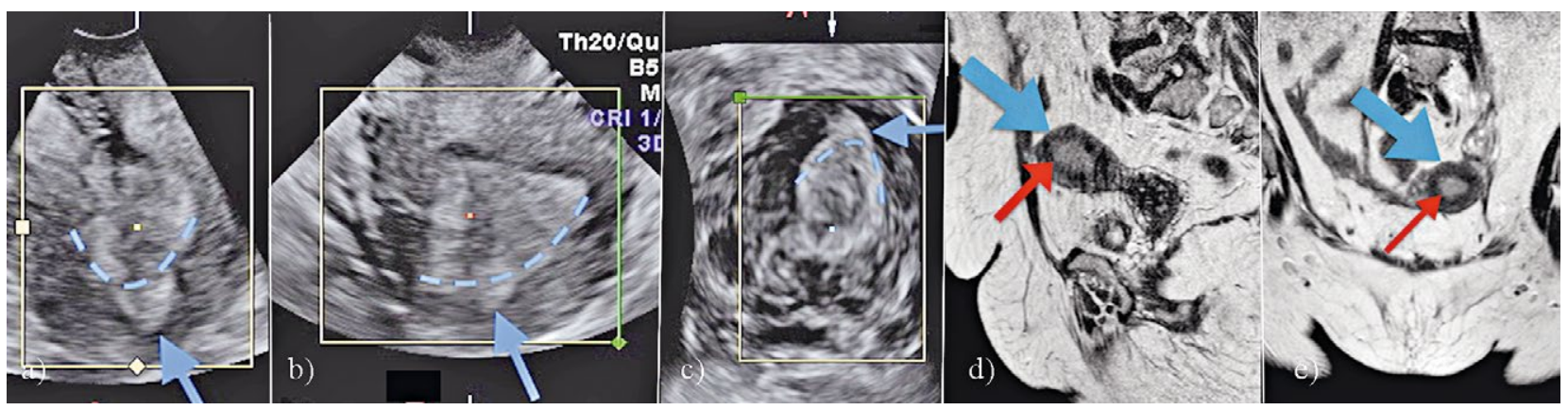

Fig 2. A patient with endometrial cancer with fundal myometrial invasion more than 50\%: 3-dimensional sonographic view of [a) coronal, b) sagittal, c) transverse view of uterus; blue arrow indicates tumoral invasion. Blue dashed line indicates the expected localization of endometrial-myometrial junction] and T2-weighted magnetic resonance images [d) sagittal, e) coronal view; Blue thick arrow indicates the uterus. Red thin line indicates the localization of the endometrial-myometrial junction]. MRI reported myometrial invasion less than 50\%. Final pathology: $75 \%$ myometrial invasion.

In figures 1 and 2 are exemplified the US and MRI findings in 2 cases included in study.

\section{Statistical Analysis}

Sensitivity, specificity, negative and positive predictive values and accuracies were calculated for 3DUS, MRI and combination of both techniques in detecting deep myometrial, lower uterine segment and cervical invasion in endometrial cancer. SPSS v17.0 (SPSS Inc. Chicago, IL, USA) was used for statistical analysis.

\section{Results}

Forty patients diagnosed with endometrial cancer were included. Demographic and clinic-pathologic variables were summarized in Table I. In all patients, the tumor was clinically confined to uterus but in 6 of them, the final pathology report revealed lymphatic metastasis. Similarly, preoperative endometrial biopsy results were endometrioid adenocarcinoma in all patients but in 3 of them, the final pathology report revealed mixed adeno- 
Table I. Demographic, clinical and pathologic data of patients

\begin{tabular}{ll}
\hline Number of patients & $\mathbf{4 0}$ \\
\hline Age (years) & $61.1 \pm 9.2$ \\
Menopause & $36(90)$ \\
FIGO stage & $17(42.5)$ \\
$\quad$ Ia & $9(22.5)$ \\
Ib & $8(20)$ \\
2 & $6(15)$ \\
3 & \\
Grade & $21(52.5)$ \\
$\quad$ I & $13(32.5)$ \\
II & $6(15)$ \\
III & \\
Histology & $37(92.5)$ \\
$\quad$ Endometrioid & $3(7.5)$ \\
$\quad$ Mixed & $17(42.5)$ \\
LVSI & $18(45)$ \\
Myometrial invasion $\geq 50 \%$ & $21(52.5)$ \\
LUSI & $11(27.5)$ \\
Cervical invasion &
\end{tabular}

Age is expressed in years \pm standard deviation, Other parameters were expressed in the number of patients(\%); LVSI - lymphovascular space invasion; LUSI - lower uterine segment invasion

Table II. The distribution of patients according to the myometrial invasion in the final histology report and different imaging modalities

\begin{tabular}{clll}
\hline \multirow{2}{*}{$\begin{array}{c}\text { Myometrial invasion } \\
\text { in imaging modality }\end{array}$} & \multicolumn{3}{c}{ Myometrial invasion in final histology } \\
\cline { 2 - 4 } & $\mathbf{2 5 0 \%}$ & $\mathbf{2 5 0 \%}$ & $\mathbf{n}$ \\
\hline 3D USG & & & \\
$\quad<50 \%$ & 19 & 2 & 21 \\
$\geq 50 \%$ & 3 & 16 & 19 \\
$\mathrm{n}$ & 22 & 18 & 40 \\
MRI & & & \\
$<50 \%$ & 16 & 4 & 20 \\
$\geq 50 \%$ & 6 & 14 & 20 \\
$\mathrm{n}$ & 22 & 18 & 40 \\
$3 \mathrm{D}$ USG & & & \\
$<50 \%$ & 14 & 1 & 15 \\
$\geq 50 \%$ & 8 & 17 & 25 \\
$\mathrm{n}$ & 22 & 18 & 40 \\
\hline
\end{tabular}

*If at least one of the techniques pointed out $\geq 50 \%$ myometrial invasion, it was considered as $\geq 50 \%$; $n$ - number of patients; $3 \mathrm{D}$ USG - three dimensional ultrasonography; MRI - magnetic resonance imaging.

carcinoma (mucinous and endometrioid). Table II represents the distribution of patients according to the myometrial invasion in the final histology report and different imaging modalities and table III represents the distribution of patients according to the lower uterine segment and cervical invasion.
Table III. The distribution of patients according to the lower uterine segment and cervical invasion in the final histology report and different imaging modalities

\begin{tabular}{clll}
\hline $\begin{array}{l}\text { LUSI in imaging } \\
\text { modality }\end{array}$ & \multicolumn{2}{l}{ LUSI in final histology } \\
\cline { 2 - 4 } & $(+)$ & $(-)$ & n \\
\hline 3D USG & & 0 & 13 \\
$(+)$ & 13 & 19 & 27 \\
$(-)$ & 8 & 19 & 40 \\
n & 21 & & \\
MRI & & 6 & 19 \\
$\quad(+)$ & 13 & 13 & 21 \\
$\quad(-)$ & 8 & 19 & 40 \\
n & 21 & & \\
3D USG + MRI* & & 6 & 23 \\
$\quad(+)$ & 17 & 13 & 17 \\
$(-)$ & 4 & 19 & 40 \\
n & 21 &
\end{tabular}

Cervical invasion in Cervical invasion in final histology

\begin{tabular}{llll}
\cline { 2 - 4 } imaging modality & $(+)$ & $(-)$ & n \\
\hline 3D USG & &
\end{tabular}

\begin{tabular}{clll}
$(+)$ & 6 & 1 & 7 \\
$(-)$ & 5 & 28 & 33 \\
$\mathrm{n}$ & 11 & 29 & 40 \\
MRI & & & \\
$(+)$ & 2 & 3 & 5 \\
$(-)$ & 9 & 26 & 35 \\
$\mathrm{n}$ & 11 & 29 & 40 \\
3D USG + MRI** & & & \\
$(+)$ & 7 & 4 & 11 \\
$(-)$ & 4 & 25 & 29 \\
$\mathrm{n}$ & 11 & 29 & 40 \\
\hline
\end{tabular}

*If at least one of the techniques pointed out lower uterine segment invasion, it was considered as positive; ** If at least one of the techniques pointed out cervical invasion, it was considered as positive; LUSI - Lower Uterine Segment Invasion, $\mathrm{n}$ - number of patients

The diagnostic performances of 3D-US in detecting deep myometrial invasion in endometrial cancer were higher compared with MRI (Table IV). The sensitivity increased when the two techniques were combined (accepted positive if at least one of them revealed deep invasion) but there was a decline in specificity and accuracy with respect to 3D-US alone. For lower uterine segment invasion, 3D-US had a similar sensitivity but higher specificity, negative and positive predictive values and accuracy than MRI. The combination of two techniques increased only the sensitivity, but the combination had a lower specificity, negative and positive predictive values and accuracy than 3D-US alone. For cervical invasion, 3D-US had better diagnostic performances than MRI in terms of these parameters mentioned above. Combination of two techniques only increased the negative predictive value. 
Predictability of myometrial, lower uterine segment and cervical invasion

Table IV. Diagnostic performances of different imaging techniques in myometrial, lower uterine segment and cervical invasion in endometrial cancer patients

\begin{tabular}{llllll}
\hline & Sensitivity & Specificity & NPV & PPV & Accuracy \\
\hline Myometrial invasion & & & & & \\
\hline 3D USG & $88.9(0.74-1)$ & $86.4(0.72-1)$ & $90.5(0.77-1)$ & $84.2(0.67-1)$ & $87.5(0.77-0.97)$ \\
MRI & $77.8(0.58-0.96)$ & $72.7(0.54-0.91)$ & $80(0.62-0.97)$ & $70(0.49-0.9)$ & $75(0.61-0.88)$ \\
3D USG + MRI & $94.4(0.83-1)$ & $63.6(0.43-0.83)$ & $93.3(0.8-1)$ & $68(0.49-0.86)$ & $77.5(0.64-0.90)$ \\
\hline Lower uterine segment invasion & $61.9(0.41-0.82)$ & $100(1-1)$ & $70.4(0.53-0.87)$ & $100(1-1)$ & $80(0.67-0.92)$ \\
3D USG & $61.9(0.41-0.82)$ & $68.4(0.47-0.89)$ & $61.9(0.41-0.82)$ & $68.4(0.47-0.89)$ & $65(0.5-0.79)$ \\
MRI & $81(0.64-0.97)$ & $68.4(0.47-0.89)$ & $76.5(0.56-0.96)$ & $73.9(0.55-0.91)$ & $75(0.61-0.88)$ \\
3D USG + MRI & & & & \\
\hline Cervical invasion & & & & \\
\hline 3D USG & $54.5(0.25-0.83)$ & $96.6(0.89-1)$ & $84.8(0.72-0.97)$ & $85.7(0.59-1)$ & $85(0.73-0.96)$ \\
MRI & $18.2(0-0.4)$ & $89.7(0.78-1)$ & $74.3(0.59-0.88)$ & $40(0-0.82)$ & $70(0.55-0.84)$ \\
3D USG + MRI & $63.6(0.35-0.92)$ & $86.2(0.73-0.98)$ & $86.2(0.73-0.98)$ & $63.6(0.35-0.92)$ & $80(0.67-0.92)$ \\
\hline
\end{tabular}

The results are expressed in $\%(95 \% \mathrm{CI})$; NPV - negative predictive value; PPV - positive predictive value; $\mathrm{CI}$ - Confidence interval; $3 \mathrm{D}$ $\mathrm{USG}$ - three dimensional ultrasonography; MRI - magnetic resonance imaging.

\section{Discussions}

Although lymphadenectomy is a part of a staging procedure in endometrial cancer, it is known that it has no survival benefit in the early stages [2,3]. From this point of view, preoperative evaluation for detecting early stage patients has became increasingly popular in order to prevent early and late complications of lymph node dissection.

One of the first studies regarding 3D-US and depth of invasion in endometrial cancer was reported in 2009. In this study, 96 patients were included [16]. The authors concluded that subjective impression of depth invasion had a sensitivity of $92.6 \%$, specificity of $82.3 \%$, negative predictive value of $96.6 \%$ and a positive predictive value of $67.7 \%$. Ergenoglu et al reported in 45 patients that subjective impression had a sensitivity of $100 \%$, a specificity of $88 \%$, a positive predictive value of $69 \%$ and a negative predictive value of $100 \%$ [12].

Some doubt may be cast on the authenticity of the evaluation with subjective impression. In the study by Mascillini et al the subjective assessment and the objective measurement techniques such as endometrial thickness, tumor/uterine anteroposterior (AP) diameter ratio, minimal tumor-free margin, minimal tumor-free margin/ uterine AP diameter ratio, tumor volume (3D), tumor/ uterine volume (3D) ratio and distance from outer cervical ostium to the lower margin of tumor were compared. They concluded that the subjective assessment of the cervical and myometrial invasion was as good as or better than any other objective measurement techniques [17].

Up to now, there are three studies that have compared 3D-US and MRI for detecting deep myometrial invasion in endometrial cancer. Our study is the first prospective study that has compared both imaging techniques for detecting deep myometrial, lower uterine segment and cervical invasion. Saarelainen et al published the first study in which they compared both techniques for detecting only deep myometrial invasion in 20 endometrial cancer patients [14]. They reported that sensitivity and negative predictive value of MRI was superior, where the specificity and positive predictive value of 3D-US was higher. In the second study, Christensen et al evaluated 61 patients with endometrial cancer and 49 patients with hyperplasia with atypia [13]. They compared 2D-US, 3D-US and MRI for both cervical and deep myometrial invasion, but MRI was performed in only $12 \%$ of patients. They found that MRI had a more diagnostic accuracy than 2DUS and 3D-US for myometrial invasion with the rates $84 \%, 75 \%$ and $71 \%$, respectively. For cervical invasion, all three imaging techniques had similar accuracy and specificity but MRI seemed to be more sensitive than the others. The last study in this group was reported by Rodríguez-Trujillo et al [15] in 98 early stage endometrial cancer patients with different histological subtypes. They found that although 3D-US was a slightly higher sensitivity than MRI $(77 \%$ vs. $69 \%)$, they had similar specificity and accuracy $(83 \%$ vs. $86 \%$ and $81 \%$ vs. $80 \%$, respectively). The authors concluded that the combination of two-techniques provided lower false negative and false positive rates.

In contrast to similar studies mentioned above, we demonstrated that 3D-US had better results compared with MRI in detecting all deep myometrial, lower uterine segment and cervical invasion. US is much more cheaper and faster technique but it requires experience. In 3D-US, 
there is the possibility to scan the uterus more than once if the quality of the obtained image is low, to store the 3D image, and evaluate it later. With an offline evaluation program, it is possible to evaluate the image repeatedly or by a different physician at a distance. Some factors were reported that influence the accuracy of the subjective assessment of tumoral invasion in endometrial cancer: tumor size, density of tumor vascularization, tumor vessel architecture and histological grading [18]. When we re-analyzed misinterpreted images in our study, in most of the patients with false negative and false positive results in the 3D-US group the myometrial invasion in final histology was between $40-60 \%$. So, misinterpretation in subjective evaluation might be due to myometrial invasion close to $50 \%$ in final histology. One patient with false negative result had fibroids and one patient with false positive result had adenomyosis. False positive rates of 3D-US for detecting both LUSI and cervical invasion were very low; but in contrast, the false negative rate was 8/21 (38\%) for LUSI and 5/11 (45\%) for cervical invasion. One of the most important reasons for false high false negative rates in our study was hypertrophy of the cervix with/without nabothian cysts in multiparous women. Other reasons were adenomyosis and fibroids.

MRI is an expensive and more demanding procedure that could easily be affected by patient related factors such as bowel or abdominal wall movements. Additionally, anatomic factors such as the presence of fibroids or adenomyoma, diffuse polypoid tumors that significantly thinned myometrium may cause misinterpretation [19]. In our study, the most important reasons for false negative and positive reasons were obesity and anatomic distortions such as myoma and adenomyosis. The highest false negative rate in MRI category was in the assessment of the cervical invasion. In this group most of the patients were obese and had nabothian cysts in the cervix.

The combination of two imaging techniques increased the sensitivity of detecting all parameters related with tumoral invasion but decreased specificity and the accuracy. Thus, the implementation of two imaging techniques is not recommended according to our study.

This study also has some limitations. Studies with larger volumes will give better results. Additionally, vascular Doppler indices may also be evaluated with a 3D-US assessment as a different study group in order to identify its performance in endometrial cancer in further trials. Since the technology in imaging techniques is improving, the newest machines with higher quality images will better identify the tumoral extension. Lastly, studies including different MRI sequences such as dynamic and diffusion-weighted images are necessary to decide which technique is better.

\section{Conclusions}

According to the results of this study, 3D-US evidenced a better performance for detecting deep myometrial, lower uterine segment and cervical invasion in endometrial cancer than MRI. 3D-US was an easier, cheap and effective imaging modality for triaging the early stage endometrial cancer patients in terms of low and high risk. Further studies with larger volumes will better elucidate us in this topic.

\section{Acknowledgements}

This study was supported by the DokuzEylul University, Scientific Research Project Fund number 2013/9.

\section{Conflict of interest: none}

\section{References}

1. Ferlay J, Autier P, Boniol M, Heanue M, Colombet M, Boyle P. Estimates of the cancer incidence and mortality in Europe in 2006. Ann Oncol 2007;18:581-592.

2. Benedetti Panici P, Basile S, Maneschi F, et al. Systematic pelvic lymphadenectomy vs. no lymphadenectomy in early stage endometrial carcinoma: a randomized clinical trial. J Natl Cancer Inst 2008;100:1707-1716.

3. ASTEC study group, Kitchener H, Swart AM, Qian Q, Amos C, Parmar MK. Efficacy of systematic pelvic lymphadenectomy in endometrial cancer (MRC ASTEC trial): a randomized study. Lancet 2009;373:125-136.

4. Vargas R, Rauh-Hain JA, Clemmer J, et al. Tumor size, depth of invasion, and histologic grade as prognostic factors of lymph node involvement in endometrial cancer: a SEER analysis. Gynecol Oncol 2014;133:216-220.

5. Kong TW, Chang SJ, Paek J, Lee Y, Chun M, Ryu HS. Risk group criteria for tailoring adjuvant treatment in patients with endometrial cancer: a validation study of the Gynecologic Oncology Group criteria. J Gynecol Oncol 2015;26:32-39.

6. Pecorelli S. Revised FIGO staging for carcinoma of the vulva, cervix, and endometrium. Int J Gynaecol Obstet 2009; 105:103-104.

7. AlHilli MM, Podratz KC, Dowdy SC, et al. Risk-scoring system for the individualized prediction of lymphatic dissemination in patients with endometrioid endometrial cancer. Gynecol Oncol 2013;131:103-108.

8. Lavie O, Uriev L, Gdalevich M, et al. The outcome of patients with stage I endometrial cancer involving the lower uterine segment. Int J Gynecol Cancer 2008;18:1079-1083.

9. Madom LM, Brown AK, Lui F, Moore RG, Granai CO, Disilvestro PA. Lower uterine segment involvement as a predictor for lymph node spread in endometrial carcinoma. Gynecol Oncol 2007;107:75-78. 
10. Dilek S, Dede M, Gezginç K, et al. Does the localisation of tumour at stage I endometrial endometrioid adenocarcinoma have an impact on invasion of the tumour and individualisation of the surgical procedure? Eur J Gynaecol Oncol 2008;29:138-1340.

11. Alcázar JL, Gastón B, Navarro B, Salas R, Aranda J, Guerriero $\mathrm{S}$. Transvaginal ultrasound versus magnetic resonance imaging for preoperative assessment of myometrial infiltration in patients with endometrial cancer: a systematic review and meta-analysis. J Gynecol Oncol 2017;28:e86.

12. Ergenoglu M, Akman L, Terek MC, et al. The prediction of myometrial infiltration by three-dimensional ultrasonography in patients with endometrial carcinoma: a validation study from Ege University Hospital. Med Ultrason 2016;18:201-206.

13. Christensen JW, Dueholm M, Hansen ES, Marinovskij E, Lundorf E, Ørtoft G. Assessment of myometrial invasion in endometrial cancer using three-dimensional ultrasound and magnetic resonance imaging. Acta Obstet Gynecol Scand 2016;95:55-64.

14. Saarelainen SK, Kööbi L, Järvenpää R, Laurila M, Mäenpää JU. The preoperative assessment of deep myometrial invasion by three-dimensional ultrasound versus MRI in endometrial carcinoma. Acta Obstet Gynecol Scand 2012;91:983-990.

15. Rodríguez-Trujillo A, Martínez-Serrano MJ, MartínezRomán S, et al. Preoperative Assessment of Myometrial Invasion in Endometrial Cancer by 3D Ultrasound and Diffusion-Weighted Magnetic Resonance Imaging: A Comparative Study. Int J Gynecol Cancer 2016;26:1105-1110.

16. Alcázar JL, Galván R, Albela S, et al. Assessing myometrial infiltration by endometrial cancer: uterine virtual navigation with three-dimensional US. Radiology 2009;250:776783.

17. Mascilini F, Testa AC, Van Holsbeke C, Ameye L, Timmerman D, Epstein E. Evaluating myometrial and cervical invasion in women with endometrial cancer: comparing subjective assessment with objective measurement techniques. Ultrasound Obstet Gynecol 2013;42:353-358.

18. Fischerova D, Frühauf F, Zikan M, et al. Factors affecting sonographic preoperative local staging of endometrial cancer. Ultrasound Obstet Gynecol 2014;43:575-585.

19. Sanjuán A, Escaramís G, Ayuso JR, et al. Role of magnetic resonance imaging and cause of pitfalls in detecting myometrial invasion and cervical involvement in endometrial cancer. Arch Gynecol Obstet 2008;278:535-539. 